\title{
Producción de artículos científicos sobre salud en seis países de América Latina, 1973 a $1992^{1}$
}

\author{
Alberto Pellegrini Filho, ${ }^{2}$ Moisés Goldbaum ${ }^{2}$ y John Silvi ${ }^{3}$
}

RESUMEN Con objeto de observar las tendencias de la investigación sobre salud en América Latina, se analizó la producción de artículos resultantes de investigaciones biomédicas, clínicas y de salud pública originadas en Argentina, Brasil, Chile, Cuba, México y Venezuela de 1973 a 1992, inclusive. De la base de datos del Institute for Scientific Information (ISI), se recuperaron 41238 artículos con primeros autores residentes en esos países. Esta producción se analizó por área de estudio, tipo de investigación, país, número de autores e instituciones que participaron en la investigación, y citaciones recibidas por cada artículo. Se hizo también un análisis de 95 artículos en epidemiología seleccionados de 570 publicados por autores de los seis países en 11 revistas de salud pública de gran prestigio internacional.

Los resultados muestran que el número de trabajos publicados aumentó $117 \%$ entre el primero y último quinquenios. La investigación clínica tuvo la distribución más homogénea entre los países y la de salud pública, la más concentrada (60,7\% se originó en el Brasil). Los artículos de investigación biomédica y de salud pública tuvieron un crecimiento relativamente mayor que los de investigación clínica a lo largo del período. Se observó una disminución relativa de artículos de un solo autor -lo que sugiere trabajo en equipo más frecuente- - y un aumento de artículos con autores vinculados a dos o más instituciones nacionales o extranjeras, lo cual indica mayor cooperación entre instituciones y países. El promedio de citaciones recibidas por artículo fue 3, lo que corresponde a menos de la mitad de las que reciben los artículos de la base del ISI $(7,78)$. En cuanto al subconjunto de los 95 artículos de epidemiología, la gran mayoría (96\%) trataban de enfermedades infecciosas o del área maternoinfantil, mientras que en la literatura internacional $78 \%$ de los artículos estaban dedicados a las enfermedades crónicas. En este conjunto se observó más cooperación con instituciones extranjeras y un indice de 4,36 citaciones por artículo.

Se concluye que, pese a las limitaciones inherentes a este tipo de estudio, se pudieron notar algunas tendencias generales del desarrollo de la investigación en los seis países con mayor producción científica de América Latina y formular hipótesis sobre los factores que determinan esas tendencias. Tomados con cautela, los resultados de estudios de este tipo pueden ser de gran valor al definir politicas de ciencia y tecnología en salud.

Próximamente en esta revista se publicará una versión en inglés de este artículo.

2 Programa de Coordinación de Investigaciones, División de Salud y Desarrollo Humano, Organización Panamericana de la Salud. Dirección postal: 525 Twenty-third Street, Washington DC 20037, Estados Unidos de América.

3 Departamento de Medicina Preventiva y Social, Facultad de Medicina de la Universidad de São Paulo, Brasil.
En este artículo se describen y analizan algunas tendencias de investigación, según se reflejan en la producción de trabajos científicos sobre la salud en Argentina, Brasil, Chile, Cuba, México y Venezuela durante el período de 1973 a 1992. Los análisis que se presentan están basados en artículos publicados en revistas de alcance internacional por autores residentes en esos seis países de América Latina durante el período considerado. En estudios anteriores $(1,2)$, las tendencias se analizaron a partir de proyectos en curso y diversas bases de datos, incluido un análisis preliminar de la producción registrada en el Institute for Scientific Information (ISI) de 
Filadefia, Pensilvania, para el período de 1972 a 1982. El presente estudio se basó en trabajos recogidos en revistas de alcance internacional indizadas por el ISI en su base de datos.

\section{TIPO DE ESTUDIO}

El tipo de estudio que nos ocupa forma parte de la cienciometría, disciplina desarrollada a principios de los años sesenta por sociólogos e historiadores de la ciencia interesados en la posibilidad de hacer análisis cuantitativos en este campo. A mediados del decenio de 1970, la preocupación por ahorrar gastos de investigación y la necesidad de evaluar sus resultados llevó a los planificadores de actividades en ciencia y tecnología a reconocer el potencial de los estudios de cienciometría para apoyar la planificación, hasta entonces basada casi exclusivamente en las opiniones de expertos.

No obstante su reconocido potencial, esos estudios han sido objeto de crítica en cuanto a sus bases conceptuales y metodológicas (3). En lo que se refiere a las primeras, se ha criticado la cienciometría por concebir la ciencia según un modelo insumo-producto $\mathrm{y}$ dedicarse solamente a medir los insumos y productos de la actividad científica, sin interesarse por analizarla como un proceso que recibe la influencia de factores intrínsecos y extrínsecos y que a la vez influye en ellos. El artículo publicado en una de las principales revistas de amplia influencia es prácticamente el único producto de la investigación científica reconocido por esos estudios.

En lo que respecta a aspectos metodológicos, se suele criticar la falta de parámetros y de criterios para evaluar la producción y productividad. Los estudios por lo general se limitan a hacer comparaciones entre países o a analizar series históricas de un mismo país para detectar tendencias. Las comparaciones entre países se critican por no tener en cuenta las diferencias culturales en materia de publicación, así como se critican las comparaciones entre producción y productividad de diferentes áreas y tipos de investigación (básica y aplicada) con patrones de publicación diferentes. Asimismo, no todos los artículos son iguales, ni representan esfuerzos equivalentes para poder ser comparados sin tener en cuenta esas diferencias.

El índice de citaciones utilizado con frecuencia en estos estudios, parece ser el caso típico de un indicador establecido antes de que se haya definido a priori qué es lo que debe indicar: calidad, impacto, u otra cualidad. Por lo general, no hay cómo eliminar las distorsiones creadas por autocitas, citas por "amiguismo" entre miembros de grupos, citas indirectas, citas negativas, etc. La fuente más utilizada para esos estudios, la base de datos del ISI, no se considera apropiada para analizar el trabajo científico de países en desarrollo, ya que incluye solamente artículos publicados por autores de esos países en las revistas más importantes del mundo industrializado. La base del ISI muestra también la tendencia a privilegiar las publicaciones en inglés.

Para superar estas limitaciones, habrá que satisfacer varias necesidades metodológicas: combinar enfoques cuantitativos y cualitativos, desarrollar bases locales para el estudio de la producción científica en países en desarrollo, y establecer nuevos indicadores que respondan a preguntas bien definidas y tengan en cuenta las diversidades entre áreas, disciplinas, tipos de investigación y otros aspectos.

Frente a las críticas de los estudios de cienciometría sería posible escoger entre dos posiciones antagónicas: abandonar de una vez este tipo de estudios por su poca utilidad, hasta contar con metodologías apropiadas; o seguir haciéndolos, entendiendo sus limitaciones y usando los resultados con cautela en la definición de políticas. Nos asociamos a esta segunda posición, en la cual se ubica el presente estudio. Reconocemos el nivel superficial y parcial del mismo, y a la vez su potencial para ayudar a entender y a describir algo de la evolución experimentada por la investigación en salud en la Región.

\section{PERÍODO DE ESTUDIO}

El período considerado, 1973 a 1992, es relativamente largo para estudios de este tipo, lo que permite observar con mayor claridad algunos aspectos del desarrollo de la actividad científica en salud en los seis países indicados. Se trata de un período más bien singular en la historia de la Región, durante el cual la producción científica manifestó un impulso notable en calidad y cantidad, y se consolidó el reconocimiento de la importancia de la ciencia y la tecnología para el desarrollo economicosocial. Este reconocimiento se manifestó a principios del período estudiado mediante la creación de organismos de planificación del desarrollo de la ciencia y la tecnología en la mayoría de los países. En aquellos que ya contaban con esos organismos, su denominación cambió de "consejos de investigación" a "consejos de desarrollo científico-tecnológico", señalando así un nuevo y más amplio ámbito de actuación. Durante ese período ocurrieron importantes transformaciones económicas, sociales, políticas e institucionales. Hubo crisis, ajustes y reanudación del crecimiento de la economía, así como deterioro y, por último, recuperación de los regímenes democráticos. Todos estos macroprocesos a la larga han repercutido en el desarrollo de la ciencia y la tecnología en la Región y, a manera de hipótesis, pueden relacionarse con algunos resultados de este estudio. ${ }^{4}$

\footnotetext{
4 A pesar de que existen varias corrientes internalistas y externalistas en perpetuo debate (4), predomina el reconocimiento de que en la dinámica de desarrollo de la actividad científica influyen tanto factores intrínsecos como extrínsecos (5). Entre los primeros están los factores de naturaleza lógicocognoscitiva, a los cuales se debe que en cada etapa histórica el progreso ulterior de la ciencia esté condicionado por el material cognoscitivo acumulado durante el período precedente. Entre los de naturaleza extrínseca se cuentan los factores relacionados con la vida material de las sociedades, como los niveles de desarrollo socioeconómico, educación y cultura de la población y otros. Para analizar con mayor precisión el grado de influencia que ejerce cada uno de esos factores, es preciso realizar estudios más profundos, diseñados con ese fin. El presente estudio, en cambio, pretende describir tendencias generales y formular algunas hipótesis sobre factores que posiblemente guarden relación con aquellas.
} 
En el mismo período se consolidó también una "desmedicalización" de la investigación en salud. Es decir, esta dejó de ser realizada básicamente por médicos en el ámbito de los servicios de salud y se diversificó en cuanto a problemas investigados, disciplinas, enfoques, tipos de investigación, formación profesional de los investigadores y ámbito institucional donde se llevaron a cabo.

Entre 1995 y 1996, la situación de la ciencia y la tecnología en salud ha presentado una serie de contradicciones. Por un lado, hay una fuerte demanda de nuevos conocimientos que ayuden a comprender la compleja situación de salud y apoyen los procesos de reforma del sector de la salud, ya en curso en la mayor parte de los países de la Región. Al mismo tiempo, el dinámico desarrollo del área biomédica exige que los grupos activos en ese campo hagan un enorme esfuerzo para mantener al día su dominio de los conocimientos y tecnologías que son de importancia estratégica, tanto para resolver problemas de salud como para promover otros sectores donde esas tecnologías puedan ser aplicadas. Por otro lado, justamente en el momento en que estas necesidades se acentúan, se observa una importante disminución de los recursos para investigación en varios países. Esto, en gran medida, se produce como consecuencia de los recortes de los gastos públicos, principal fuente de recursos para la investigación en salud.

Se reconoce ampliamente la acuciante necesidad de que el Estado no se limite a su rol de financiador y ejecutor de investigaciones y amplíe su función reguladora de la actividad científicotecnológica, liderando el proceso de convocar a los diversos actores involucrados en esas actividades para lograr una definición concertada de los rumbos por seguirse. Sin embargo, vemos por el contrario que los consejos de ciencia y tecnología, órganos gubernamentales encargados de asumir dichas responsabilidades en los países de la Región, están sufriendo reducciones de sus recursos humanos y financieros y de su fuerza política.
Este estudio forma parte de un conjunto de actividades de la OPS cuyo propósito es cooperar con los países de la Región en la superación de esos desafíos y contradicciones. Específicamente, busca llamar la atención hacia algunas de las características de la producción científica durante el período considerado, entendiendo que puede así contribuir a un mejor análisis de la situación y sus tendencias y, por lo tanto, a una fundamentación más acertada de las políticas de ciencia y tecnología en salud.

\section{METODOLOGÍA}

En el presente trabajo se utilizó la base de datos del ISI. La base es bastante selectiva e incluye cerca de 3500 revistas, de las más importantes en los diversos campos de la ciencia. Según su creador, E. Garfield, esta cobertura selectiva no obedece solamente a razones económicas, sino al hecho de que en cualquier campo es relativamente reducido el número de revistas realmente importantes, más frecuentemente leídas y citadas, fenómeno este conocido como la "ley de Bradford" (6). En la propia base del ISI, ya bastante selectiva, más de $50 \%$ de los artículos y $80 \%$ de las citaciones figuran en solamente 500 revistas. Esta base permite, por lo tanto, no un inventario de toda la producción científica de los países considerados, sino de la parte de la producción que circula en la literatura internacional considerada por los responsables de esta base como la de mayor calidad. ${ }^{5}$

La base del ISI utilizada, ISI Database Science Indicators File, incluye el Science Citation Index, el Social Sciences Citation Index y el Arts and Humanities Citation Index. Para cubrir todos los campos de la ciencia, esta base utiliza 89 Category Codes o CatCodes [códigos de categorías], de los cuales seleccionamos 38 para delimitar el campo de las ciencias

\footnotetext{
5 Los países de América Latina y el Caribe que en los años setenta aportaban cerca de $1 \%$ de los artículos a esa base, aumentaron su participación a $1,5 \%$ durante los años ochenta (6).
}

de la salud humana. Posteriormente, subdividimos este conjunto en investigación biomédica, clínica y de salud pública (7). Cada uno de los códigos incluye un grupo de revistas periódicas y se presume que todos los artículos publicados en ellas pertenecen a ese código. En el período de referencia, los 38 códigos incluyeron 1975 revistas, de las que se tomaron los artículos analizados en el presente estudio.

Los seis países seleccionados -Argentina, Brasil, Chile, Cuba, México y Venezuela- son responsables de cerca de $90 \%$ de toda la producción científica de América Latina y el Caribe, tanto en lo que se refiere a la ciencia en general, como al campo de las ciencias de la salud en particular (6). Cabe notar que, en el caso de Cuba, la producción científica registrada en la base está superada en términos cuantitativos por otros cuatro países no incluidos en este estudio (Colombia, Jamaica, Perú y Uruguay); sin embargo, su inclusión se justifica por las características particulares de las políticas de ciencia y tecnología en ese país. ${ }^{6}$ En el total de 20 años considerados, 32 títulos de revistas de salud de América Latina (29 de los seis países) fueron incluidas en la base del ISI, en el que figuran anualmente entre $17 \mathrm{y}$ 21 de ellas (anexo 1).

Para todo el período de 1972 a 1992, se obtuvieron 72899 registros con primeros autores que residían en alguno de los seis países. Se decidió trabajar solamente con artículos de investigación, excluyéndose editoriales, notas, cartas, revisiones, resúmenes de reuniones, etc., lo que hizo que el número de registros se redujera a 42292 trabajos. El primer año de la base, 1972, fue excluido por razones de consistencia y uniformidad. Eso redujo los artículos a un total de 41238.

El registro de cada artículo incluye año de publicación, título del artículo, nombre de los autores con sus respecti-

\footnotetext{
6 Durante el período analizado se consolidó en Cuba un modelo de planificación de la ciencia y la tecnología basado en los problemas de varios niveles (nacionales, sectoriales, locales, etc.) y definidos por los organismos estatales correspondientes bajo la coordinación de la Academia de Ciencias.
} 
CUADRO 1. Número de artículos publicados por quinquenio en cada país, según el tipo de investigación, 1973-1992

\begin{tabular}{|c|c|c|c|c|c|c|}
\hline País & $\begin{array}{l}\text { Tipo de } \\
\text { estudio }\end{array}$ & $1973-1977$ & $1978-1982$ & $1983-1987$ & 1988-1992 & 1973-1992 \\
\hline Argentina & $\begin{array}{l}\text { Biomédica } \\
\text { Clínica }\end{array}$ & $\begin{array}{l}1028 \\
1052\end{array}$ & $\begin{array}{l}1050 \\
1576\end{array}$ & $\begin{array}{l}1622 \\
1687\end{array}$ & $\begin{array}{l}1668 \\
1751\end{array}$ & $\begin{array}{l}5368 \\
6066\end{array}$ \\
\hline Brasil & $\begin{array}{l}\text { Biomédica } \\
\text { Clínica } \\
\text { Salud pública }\end{array}$ & $\begin{array}{r}749 \\
628 \\
47\end{array}$ & $\begin{array}{r}1366 \\
1540 \\
80\end{array}$ & $\begin{array}{r}1946 \\
1615 \\
264\end{array}$ & $\begin{array}{r}2947 \\
2085 \\
281\end{array}$ & $\begin{array}{r}7008 \\
5868 \\
672\end{array}$ \\
\hline Cuba & $\begin{array}{l}\text { Biomédica } \\
\text { Clínica } \\
\text { Salud pública }\end{array}$ & $\begin{array}{r}37 \\
43 \\
3\end{array}$ & $\begin{array}{r}59 \\
77 \\
0\end{array}$ & $\begin{array}{r}97 \\
75 \\
2\end{array}$ & $\begin{array}{r}145 \\
94 \\
7\end{array}$ & $\begin{array}{r}338 \\
289 \\
12\end{array}$ \\
\hline México & $\begin{array}{l}\text { Biomédica } \\
\text { Clínica } \\
\text { Salud pública }\end{array}$ & $\begin{array}{r}289 \\
1012 \\
23\end{array}$ & $\begin{array}{r}446 \\
1264 \\
30\end{array}$ & $\begin{array}{r}669 \\
1392 \\
31\end{array}$ & $\begin{array}{r}905 \\
1372 \\
40\end{array}$ & $\begin{array}{r}2309 \\
5040 \\
124\end{array}$ \\
\hline \multirow[t]{2}{*}{ Todos } & $\begin{array}{l}\text { Biomédica } \\
\text { Clínica } \\
\text { Salud pública }\end{array}$ & $\begin{array}{r}2555 \\
3673 \\
149\end{array}$ & $\begin{array}{r}3714 \\
5561 \\
142\end{array}$ & $\begin{array}{r}5218 \\
5971 \\
387\end{array}$ & $\begin{array}{r}6621 \\
6818 \\
429\end{array}$ & $\begin{array}{r}18108 \\
22023 \\
1107\end{array}$ \\
\hline & Total & 6377 & 9417 & 11576 & 13868 & 41238 \\
\hline
\end{tabular}

vas instituciones y países de residencia, revista en que fue publicado y, para los artículos publicados a partir de 1982, el número de citaciones recibidas en cada año subsecuente a su publicación.

El análisis que sigue debe ser considerado como una primera aproximación, que se limita a identificar las grandes tendencias de carácter general para todo el campo de la investigación en salud en el período de 20 años. Sin embargo, con miras a explorar las posibilidades de un estudio en mayor profundidad, se abordaron también las tendencias de la producción científica en la epidemiología. De cerca de 40 revistas de investigación en salud pública incluidas en la base de datos, escogimos las 11 revistas $^{7}$ internacio-

\footnotetext{
7 Las 11 revistas seleccionadas son: American Journal of Epidemiology, American Journal of Public Health, American Journal of Tropical Medicine and Hygiene, Bulletin of the World Health Organization, Epidemiology and Infection, International Journal of Epidemiology, Journal of Clinical Epidemiology, Medical Care, Preventive Medicine, Public Health Report y Transactions of the Royal Society of Tropical Medicine and Hygiene.
}

nales que consideramos de mayor importancia. Se revisaron todos los registros de los 570 artículos publicados por esas revistas entre 1973 y 1992, cuyo primer autor residía en uno de los seis países. De este modo se seleccionaron 95 artículos de epidemiología para un análisis más detallado.

\section{HALLAZGOS PRINCIPALES Y DISCUSIÓN}

Entre 1973 y 1992 se publicó un total de 41238 artículos de autores residentes en los seis países, de los cuales $38,3 \%$ vieron la luz en la primera década y $61,7 \%$, en la segunda (cuadro 1). Comparando la producción por quinquenios, se observa el siguiente incremento: en 1973-1977 aparecieron 6377 artículos; en 1978-1982, 9417 artículos (crecimiento de $47 \%$ en relación al quinquenio anterior); en 1983-1987, 11576 artículos (crecimiento de $23 \%$ ) y entre 1988-1992, 13868 (crecimiento de $20 \%$ ). Es interesante observar que esta evolución no está relacionada con el número de revistas de los seis países en la base del ISI, el cual se mantuvo más o menos estable y en los quinquenios especificados ascendía a 17, 21, 20 y 18 , respectivamente.

Como se puede observar, entre el primero y el último quinquenios hubo un crecimiento de $117 \%$ en el número de artículos publicados, con variaciones en el ritmo de crecimiento. Estas variaciones pueden estar relacionadas con la inestabilidad de los recursos para investigación observada en ese período. El mayor incremento en el número de artículos ocurrió entre el primero y segundo quinquenios, período que coincide con un crecimiento de los indicadores económicos y de los gastos en ciencia y tecnología para toda la Región. Posteriormente, durante el decenio de 1980 y, particularmente a partir de 1983, hubo una disminución de esos gastos en la mayor parte de los países (8) y luego una recuperación al final del decenio. 
FIGURA 1. Porcentaje de crecimiento del número de artículos sobre salud originados en seis países de América Latina, por quinquenios, de 1973 a 1992

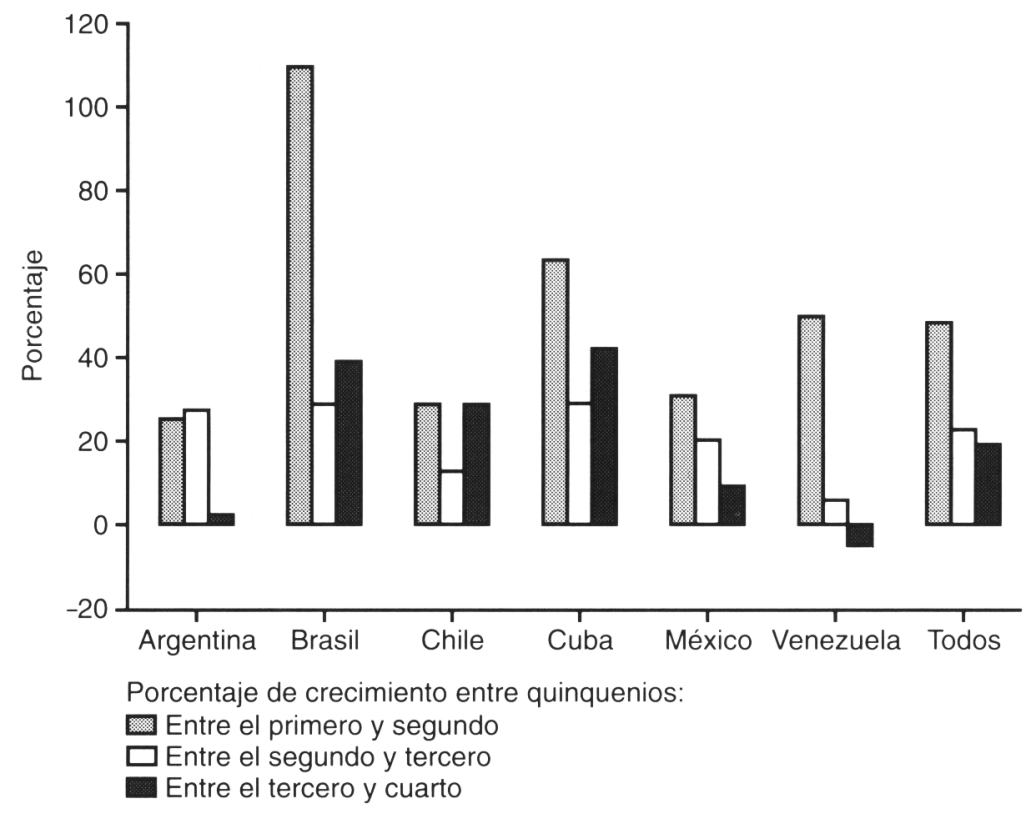

Fuente: Institute for Scientific Information, Filadelfia, Pensilvania, EUA.

\section{Datos por país}

Los datos desagregados por país (figura 1) muestran que el aumento del número de artículos no fue uniforme de un país a otro. Llama la atención el pronunciado aumento del número de artículos por autores brasileños entre el primero y segundo quinquenios $(110 \%)$, la reducción del crecimiento a $28 \%$ en el siguiente período y una leve recuperación a 39\% entre el tercero y cuarto quinquenios. Cuba y Chile siguieron un patrón similar, pero no con la misma magnitud de crecimiento entre el primero y segundo quinquenios. También se destaca la pequeña magnitud del crecimiento observado en México, Argentina y Venezuela entre el tercero y cuarto quinquenios, especialmente en el caso de Venezuela, que tuvo un incremento importante entre el primero y segundo quinquenios, el cual menguó manifiestamente en los años siguientes, hasta llegar a un crecimiento negativo entre el tercero y el cuarto períodos. Posiblemente este comportamiento esté relacionado con las políticas estrictas de ajuste económico y disminución del gasto público aplicadas en esos países a fines de los años ochenta.

Como ya se ha hecho notar, los seis países seleccionados originaron cerca de $90 \%$ de los artículos de América Latina y el Caribe registrados en el ISI. Dentro del propio grupo se notó también una concentración (61\%) de trabajos de dos países en el total de publicaciones consideradas: 28,1\% provenían Esa concentración tendió a aumentar durante el período de estudio. Si se consideran los dos años límite, 57\% de los artículos en 1973 correspondieron a la Argentina y el Brasil, cifra que llegó a $68 \%$ en 1992 .

Es interesante observar cómo ha cambiado el peso relativo de los artículos publicados por autores de la Argentina y el Brasil. La Argentina fue responsable de la mayor parte de los artículos en 1973 (38\% del total), doble en proporción a los del Brasil (19\%). Para 1992, estas posiciones se habían invertido y el Brasil figuraba con casi la mitad del total de artículos (45\%), mientras que la Argentina estaba de la Argentina y 32,9\% del Brasil. representada por $23 \%$. Ese cambio de posición empezó a notarse ya entre el primero y segundo quinquenios, cuando el Brasil pasó a aportar de 22 a $31 \%$ del total de artículos de los seis países, mientras que los de la Argentina se redujeron, de 33 a $28 \%$. Este trueque se debió tanto al notable incremento de producción observado en el Brasil entre los dos períodos (110\%), como al bajo desempeño de la Argentina (25\% de aumento), inferior al crecimiento promedio de los seis países $(48 \%)$, situación que podría estar vinculada con la inestabilidad política e institucional y la emigración de científicos de este último país a mediados de los años setenta.

\section{Producción por tipo de investigación}

El trabajo científico en los seis países tampoco fue homogéneo según nuestra clasificación por tres tipos de investigación: biomédica, clínica y de salud pública (cuadro 2). La investigación clínica, de mayor tradición en la región, se distribuyó de manera más o menos uniforme, mientras que las investigaciones biomédica y de salud pública estuvieron concentradas en gran parte en el Brasil y la Argentina, responsables de casi $70 \%$ de los artículos publicados en esas áreas. A los autores del Brasil correspondieron 60,7\% de los 1107 artículos sobre salud pública, proporción con tendencia ascendente durante el período establecido. ${ }^{8}$

La última cifra es preocupante, ya que la investigación de salud pública (es decir, sobre los sistemas y la situación de salud) debería estar más ampliamente representada en todos los países. Es un elemento fundamental para la toma de decisiones sobre

\footnotetext{
8 Esta concentración no está relacionada con el origen brasileño de las únicas dos revistas de salud pública de los seis países indizadas por el ISI entre 1972 y 1992: la Revista del Instituto de Medicina Tropical y la Revista de Salud Pública. Durante el período estudiado, estas dos revistas publicaron 951 artículos de autores de los seis países, 429 de los cuales tienen autores brasileños $(45 \%)$. Como se verá más adelante, de los artículos de epidemiología publicados en las 11 revistas internacionales seleccionadas, $66 \%$ (63/95) son del Brasil.
} 
CUADRO 2. Distribución porcentual de la publicación de artículos científicos por país, según el tipo de investigación, 1973-1992

\begin{tabular}{lccc}
\hline & \multicolumn{3}{c}{ Tipo de investigación } \\
\cline { 2 - 4 } \multicolumn{1}{c}{ País } & Biomédica & Clínica & Salud pública \\
\hline Argentina & 29,6 & 27,5 & 13,5 \\
Brasil & 38,7 & 26,6 & 60,7 \\
Chile & 11,0 & 17,2 & 9,9 \\
Cuba & 1,9 & 1,3 & 1,1 \\
México & 12,7 & 22,9 & 11,2 \\
Venezuela & 6,0 & 4,4 & 3,6 \\
\hline
\end{tabular}

Fuente: Institute for Scientific Information, Filadelfia, Pensilvania, EUA.

CUADRO 3. Distribución porcentual por tipo de investigación de artículos científicos originados en seis países de América Latina, ${ }^{a}$ de 1973 a 1992 y desglosados en dos decenios

\begin{tabular}{lccc}
\hline & \multicolumn{3}{c}{ Años } \\
\cline { 2 - 4 } Tipo de investigación & $1973-1982$ & $1983-1992$ & $1973-1992$ \\
\hline Biomédica & 39,7 & 46,5 & 43,9 \\
Clínica & 58,5 & 50,3 & 53,4 \\
Salud pública & 1,8 & 3,2 & 2,7 \\
\hline
\end{tabular}

Fuente: Institute for Scientific Information, Filadelfia, Pensilvania, EUA.

${ }^{a}$ Argentina, Brasil, Chile, Cuba, México y Venezuela.

políticas de salud frente a la gran diversidad de situaciones y problemas de salud que afectan a las poblaciones.

La distribución de los artículos por tipo de investigación (cuadro 3) muestra además que en el análisis de los problemas de salud aún prevalece el enfoque individual sobre el poblacional. Apenas 2,7\% de los artículos correspondieron a la clasificación de investigación en salud pública, en contraste con $53,4 \%$ de investigación clínica y $43,9 \%$ de biomédica. A lo largo del período hubo una tendencia hacia el crecimiento de las investigaciones en salud pública y biomédicas, y una consecuente disminución de la importancia relativa de la investigación clínica.

Esa tendencia parece acompañar, con cierto retraso temporal, las tendencias observadas a nivel de la literatura mundial. En 1982, la OPS hizo un estudio sobre las tendencias de la producción científica, comparando el contenido de la literatura mundial con el de la latinoamericana (9). Para la primera se utilizó el Index Medicus (IM) de la
National Library of Medicine [Biblioteca Nacional de Medicina] de los Estados Unidos de América, y para la segunda, el Index Medicus Latinoamericano (IMLA) del Centro Latinoamericano y del Caribe de Información en Ciencias de la Salud (BIREME). En la literatura mundial, entre 1966 y 1980, las áreas temáticas que presentaron mayor expansión (puntos de crecimiento) fueron las ciencias físicas relacionadas con la salud $(+193 \%)$, las ciencias biológicas $(+143 \%)$, y la salud pública $(+148 \%)$, mientras que el crecimiento para todas las áreas en conjunto fue de $51 \%$. Comparando el perfil de la producción catalogada en el IM para 1980 y en el IMLA para 1979-1982, se observó que de las 14 categorías $\mathrm{MeSH}$ (medical subject headings) la gran mayoría de los artículos del IMLA $(42 \%)$ se incluyeron bajo "enfermedades", mientras que en el IM solamente $15 \%$ de los artículos se clasificaron en esa categoría. Esto puede significar un mayor desarrollo relativo en América Latina de la investigación clínica, o sea de las enfermedades, frente al estudio de los fenómenos biológicos básicos no directamente relacionados con ellas. De hecho, la producción en ciencias biológicas y ciencias físicas es mayor en la literatura internacional, 12 y $5 \%$ respectivamente en el IM, frente a $9 \mathrm{y}$ $1 \%$ en el IMLA.

En el estudio citado (9) se concluye que el perfil de las publicaciones latinoamericanas para el período 19791982 se asemeja más a lo que se observaba en la literatura internacional a mediados de los años sesenta que a lo que estaba ocurriendo en 1980. Lo observado en el estudio de 1983 no se puede comparar con lo que muestra el presente documento, ya que el primero analizó lo que se publicaba en las revistas de la Región y en las revistas internacionales (adjudicándole como origen a cada artículo el país donde se publicaba la revista y no el país de residencia del autor), mientras que el presente estudio analiza los patrones de publicación de los autores residentes en seis países. Sin embargo, se puede decir que la tendencia al aumento de la investigación biomédica y disminución de la investigación clínica observada en el presente estudio refleja que la producción científica de los autores de los seis países se aproxima al perfil de distribución observado en la literatura internacional.

Aunque la tendencia mencionada arriba se observa en todos los países estudiados, los análisis de las curvas de producción por áreas y quinquenios revelan particularidades interesantes (véase el cuadro 1). Así, en la Argentina, durante el primer quinquenio, las investigaciones biomédica y clínica tenían un peso prácticamente igual (48 y $49 \%$, respectivamente). En el quinquenio siguiente hubo crecimiento de la investigación clínica y estancación de la investigación biomédica, pero esta tendió a recuperarse en los períodos siguientes. Este perfil parece indicar que los acontecimientos de mediados del decenio de 1970 tuvieron un impacto negativo mayor en la investigación biomédica que en las demás.

En los casos del Brasil y Cuba, el ritmo de crecimiento de la investiga- 
ción biomédica superó al de la clínica, y el período terminó con el claro predominio de la primera. En Cuba, el crecimiento de la investigación biomédica en el último decenio fue sobresaliente, probablemente debido a los esfuerzos que invirtió el país en promover el desarrollo de la biotecnología.

Chile y México, y el segundo país en particular, tienen la más larga tradición de investigación clínica. Aunque en ambos se mantuvo ese predominio, la investigación biomédica presentó un ritmo de crecimiento discretamente mayor al de la clínica. Venezuela fue el único país en el que la investigación biomédica, predominante a comienzos del período, terminó en situación inferior a la clínica. La disminución drástica, tanto en términos relativos como absolutos, de la investigación biomédica entre el penúltimo y último quinquenios, quizá indica su vulnerabilidad frente a la crisis económica y a las políticas de ajuste implantadas en ese período.

\section{Colectivización del trabajo científico}

Un fenómeno que expresa cierta "modernización" de la actividad científica en los países estudiados es el incremento de la colectivización del trabajo, pues se observó una disminución relativa del número de artículos publicados por investigadores aislados. En la primera década del período los artículos con un solo autor ascendieron a $17 \%$, pero ese porcentaje se redujo a $10 \%$ en la segunda década, mientras que los artículos de seis o más autores pasaron de 8 a 15\% (cuadro 4).

El fenómeno de la colectivización de la actividad científica se tradujo también en una tendencia a mayor cooperación entre instituciones dentro y fuera del país. Como consecuencia, aumentó el número de artículos cuyo primer autor es de uno de los países seleccionados y los demás autores, de otras instituciones nacionales o internacionales. Así, para todo el período de 1973 a 1992, 73,4\% de los artículos fueron producidos por autores de una

\section{CUADRO 4. Distribución porcentual del número de autores por artículo en el total de trabajos originados en seis países de América Latina ${ }^{\mathrm{a}}$ durante los dos decenios indicados}

\begin{tabular}{lcc}
\hline \multirow{2}{*}{$\begin{array}{c}\text { Número de autores } \\
\text { por artículo }\end{array}$} & \multicolumn{2}{c}{ Período } \\
\cline { 2 - 3 } & $1973-1982$ & $1983-1992$ \\
\hline 1 & 25 & 10 \\
2 & 50 & 21 \\
3 a 5 & 8 & 15 \\
6 o más & 17 & \multicolumn{2}{c}{10} \\
\hline
\end{tabular}

Fuente: Institute for Scientific Information, Filadelfia, Pensilvania, EUA.

argentina, Brasil, Chile, Cuba, México y Venezuela. sola institución, mientras que $18,8 \%$ involucraron autores de instituciones nacionales diferentes de la del primer autor (cooperación interinstitucional interna) y $7,7 \%$ incluyeron autores de instituciones de otros países (cooperación interinstitucional externa). Entre el primer decenio y el segundo, disminuyeron los artículos de una sola institución de 77,6 a 70,9\% y aumentaron los de cooperación interna de 16,2 a $20,4 \%$, y los de cooperación externa de $6,3 \%$ a $8,6 \%$.

Al comparar los datos de todo el período según áreas de estudio (cuadro 5), la investigación biomédica se destaca por presentar la mayor proporción de cooperación externa $(10,1 \%$ contra $5,8 \%$ de la clínica y $8,4 \%$ de salud pública), mientras que la investigación en salud pública presenta más cooperación interna $(25,7 \%)$ que la biomédica $(18,5 \%)$ y la clínica $(18,6 \%)$. La investigación clínica presentó el mayor número de artículos relacionados con una sola institución $(75,5 \%$ contra $71,4 \%$ de los biomédicos y $65,9 \%$ de los de salud pública). Cabe recordar que estos datos sobre la cooperación interinstitucional solo se refieren a artículos cuyo primer autor vivía en uno de los países seleccionados.

\section{Origen de autores y revistas}

La producción científica de los seis países seleccionados no presenta un grado exagerado de concentración en unos pocos autores. Por el contrario, los 41238 artículos fueron producidos estos aportó solo uno (63\%) o dos (17\%) trabajos, rara vez seis o más $(6 \%)$.

En lo que se refiere al país de origen de las revistas donde publicaron los autores, se notaron algunos aspectos interesantes. Cerca de 30\% de los artículos producidos por autores residentes en Argentina, Brasil y México y registrados en la base del ISI se publicaron en revistas nacionales. En el caso de Chile, esta cifra llegó a 50\%, y a 10\% en Cuba y Venezuela. De la producción publicada en el extranjero por autores residentes en Argentina, Brasil y Chile, por 20095 autores y la mayor parte de

CUADRO 5. Distribución porcentual por tipo de investigación de los trabajos originados en seis países de América Latina, ${ }^{a}$ 1973-1992, según las instituciones involucradas

\begin{tabular}{lccr}
\hline \multirow{2}{*}{$\begin{array}{c}\text { Tipo de } \\
\text { investigación }\end{array}$} & \multicolumn{3}{c}{ Instituciones cooperantes } \\
\cline { 2 - 4 } & Una local & Varias locales & Extranjeras \\
\hline Biomédica & 71,4 & 18,5 & 10,1 \\
Clínica & 75,5 & 18,6 & 5,8 \\
Salud pública & 65,9 & 25,7 & 8,4 \\
\hline
\end{tabular}

Fuente: Institute for Scientific Information, Filadelfia, Pensilvania, EUA.

argentina, Brasil, Chile, Cuba, México y Venezuela. 
cerca de 35\% correspondió a revistas de los Estados Unidos y $60 \%$ a revistas europeas (entre estas, 20\% del Reino Unido). Las revistas de los Estados Unidos tuvieron mucha más importancia para los autores de México y Venezuela y en ellas publicaron 50 y $45 \%$ de unos y otros, respectivamente. Las revistas europeas recogieron cerca de $45 \%$ (20\% del Reino Unido) de la producción de esos dos países. Cuba presentó un patrón diferente, con cerca de $80 \%$ de publicación en revistas europeas (14\% del Reino Unido y 65\% de otros países) y apenas $14 \%$ en revistas de los Estados Unidos. En el anexo 2 aparecen listas de las revistas que publicaron el mayor número de artículos de cada país.

Llama la atención la relativamente escasa publicación en revistas de América Latina y el Caribe, lo que puede interpretarse como poco intercambio entre países de la Región, por lo menos en lo que se refiere a las revistas indizadas por el ISI. Los autores de México $(1,6 \%)$, Argentina (1,7\%), y Brasil $(2,7 \%)$ son los que menos publican en revistas latinoamericanas, y los que más publican en ellas, si bien en baja proporción, son de Cuba $(7,8 \%)$, Venezuela $(6,1 \%)$ y Chile $(5,4 \%)$.

\section{Citaciones por artículo}

Las citaciones recibidas por un artículo determinado son tradicionalmente utilizadas como medida de su impacto. Por supuesto que, así como el artículo científico no es el único producto de la actividad científica, las citaciones que recibe tampoco son la única medida de su impacto ni mucho menos de su calidad. Teniendo en cuenta las limitaciones de este indicador, pero reconociendo su amplia aceptación como medida aproximada de la acogida de un trabajo por la comunidad científica, se han analizado las citaciones recibidas por los artículos cuyo primer autor reside en uno de los seis países durante el período de 1982 a 1992.

El cociente entre el número de citaciones y el número de artículos produ- cidos en un período se utiliza como indicador de impacto de la producción científica en ese período. Según Garfield (6), de 1981 a 1993 el promedio mundial de citaciones por artículo de todas las ciencias fue 7,78, mientras que para los de América Latina y el Caribe fue de 3,52, lo que indica un impacto relativo (citaciones por artículo de América Latina y el Caribe relativas a citaciones por artículo mundial) de 0,45. En ciencias de la salud, el promedio de citaciones por artículo para los seis países fue 3,10, promedio próximo al de la Región para todas las ciencias. Buscando medir el impacto relativo en este grupo, o sea, citaciones por artículo de un país relativas a citaciones por artículo del conjunto de los seis, se obtuvieron los siguientes resultados: Argentina, 1,00; Brasil, 0,91; Chile, 0,84; Cuba, 0,50; México, 1,20 y Venezuela, 1,47.

Tampoco fue uniforme el impacto por tipo de investigación, ya que hubo diferencias importantes entre el impacto de los artículos biomédicos, clínicos y de salud pública, con gran ventaja para los primeros, ya que en promedio cada uno de ellos recibió 4,03 citaciones, los de clínica 2,29 y los de salud pública, 1,61. En términos de impacto relativo, la biomédica obtuvo 1,30; la clínica, 0,74; y la de salud pública, 0,52. De las 90995 citaciones recibidas durante el período, 60,5\% correspondieron a artículos biomédicos, 38\% a clínicos y 1,5\% a salud pública, mientras que la distribución de los artículos en esas mismas áreas fue de $46,3 \%$, 50,6\% y 3,1\%, respectivamente. Todo lo anterior indica un impacto mayor de los artículos del área biomédica. Cabe observar que la investigación en salud pública, con un porcentaje de artículos bastante inferior al de las otras dos áreas, tuvo una participación relativa aun menor en lo que se refiere a las citaciones recibidas.

\section{Producción en epidemiología}

Conforme se mencionó en la sección de metodología, para el análisis de la producción en epidemiología se seleccionaron las 11 revistas de salud pública consideradas de mayor importancia. Se trata, por lo tanto, de un análisis preliminar y parcial de la producción en epidemiología, ya que se limita a los artículos publicados en las revistas de salud pública, sin incluir artículos de epidemiología publicados ocasionalmente por revistas como Lancet, $J A M A$, etc. En las revistas seleccionadas se identificaron 95 artículos de epidemiología entre los 570 cuyos primeros autores residían en alguno de los seis países.

Los autores brasileños produjeron la gran mayoría de esos 95 artículos $(66 \%)$, grado de concentración semejante al observado en la producción total en salud pública de los seis países. En lo que se refiere al número de autores por artículo, el porcentaje de trabajos de un solo autor (31\%) fue bastante superior al que se observó para el total de trabajos, de los cuales apenas $12,8 \%$ tenían un solo autor.

No obstante lo dicho, en este conjunto de proyectos llamó la atención la importancia de la colaboración entre instituciones, extranjeras en particular, que fue muy superior a la del total de artículos. Aunque 32\% de los artículos fueron producidos por autores pertenecientes a una misma institución y 26\% a instituciones diferentes del mismo país (cooperación interna), $42 \%$ incluyeron autores de otros países (cooperación externa). Para el total de artículos, las cifras son respectivamente $73,4 \%, 18,8 \%$ y $7,7 \%$, lo cual parece indicar que los epidemiólogos que publican en estas revistas tienen mayor contacto con la comunidad científica internacional que el total de autores.

La gran mayoría de los artículos $(83 \%)$ se refieren a la epidemiología de las enfermedades infecciosas, solo $4 \%$ a enfermedades crónicas y 13\% a otros temas, sobre todo del área maternoinfantil. Para comparar este perfil temático con el de la producción mundial, se consultaron dos de las revistas consideradas más importantes: International Journal of Epidemiology y American Journal of Epidemiology. La primera de estas revistas, en 1987, 1989 y 1991 
publicó 381 artículos de los cuales $25,7 \%$ (98) estuvieron relacionados con enfermedades infecciosas y problemas maternoinfantiles. En la segunda revista, durante el segundo semestre de cada año se publicaron 344 artículos, de los cuales 19,2\% (66) correspondían a enfermedades infecciosas $y$ temas maternoinfantiles. Estos datos indican que si bien el estudio de las enfermedades crónicas predomina en la literatura epidemiológica a nivel mundial, los epidemiólogos de la Región que logran publicar en revistas de gran circulación internacional todavía se dedican a las enfermedades infecciosas. De los 381 artículos del International Journal of Epidemiology, apenas $3(0,78 \%)$ fueron publicados por autores de los seis países.
En lo que se refiere a las citaciones recibidas por los artículos de epidemiología, los 77 publicados entre 1981 y 1992 recibieron 336 citaciones, con un índice de impacto de 4,36 citaciones por artículo, bastante superior al verificado para el área de salud pública $(1,61)$, como para el total de artículos de investigación en salud de los seis países en conjunto (3).

ANEXO 1. Las 32 revistas de salud latinoamericanas incluidas en la base de datos del Institute for Scientific Information de 1972 a 1992

\begin{tabular}{|c|c|c|}
\hline País & Revista & Artículos publicados (No.) \\
\hline Argentina & $\begin{array}{l}\text { Acta Physiologica et Pharmacologica Latinoamericana } \\
\text { Acta Physiologica Latinoamericana } \\
\text { Acta Psiquiátrica y Psicológica de América Latina } \\
\text { Medicina (Buenos Aires) } \\
\text { Microscopía Electrónica y Biología Celular } \\
\text { Prensa Médica Argentina }\end{array}$ & $\begin{array}{r}238 \\
459 \\
94 \\
1312 \\
70 \\
1657\end{array}$ \\
\hline Brasil & $\begin{array}{l}\text { Arquivos Brasileiros de Psicologia } \\
\text { Arquivos Brasileiros de Psicologia Aplicada } \\
\text { Arquivos de Neuro-Psiquiatria } \\
\text { Brazilian Journal of Medical and Biological Research } \\
\text { Memórias do Instituto Oswaldo Cruz } \\
\text { Revista Brasileira de Genética } \\
\text { Revista Brasileira de Medicina } \\
\text { Revista Brasileira de Pesquisas Médicas e Biológicas } \\
\text { Revista do Instituto de Medicina Tropical de São Paulo } \\
\text { Revista de Saúde Pública }\end{array}$ & $\begin{array}{r}325 \\
42 \\
33 \\
686 \\
345 \\
726 \\
1057 \\
170 \\
584 \\
449\end{array}$ \\
\hline Chile & $\begin{array}{l}\text { Archivos de Biología y Medicina Experimentales } \\
\text { Revista Médica de Chile }\end{array}$ & $\begin{array}{r}208 \\
2759\end{array}$ \\
\hline Colombia & $\begin{array}{l}\text { Archivos de la Sociedad Americana de Oftalmología y Optometría } \\
\text { Revista Latinoamericana de Psicología }\end{array}$ & $\begin{array}{r}1 \\
97\end{array}$ \\
\hline Cuba & Interferón y Biotecnología & 73 \\
\hline México & $\begin{array}{l}\text { Archivos del Instituto de Cardiología de México } \\
\text { Archivos de Investigación Médica } \\
\text { Investigación Médica Internacional } \\
\text { Medicina (México) } \\
\text { Patología (México) } \\
\text { Revista de Investigación Clínica } \\
\text { Revista Mexicana de Radiología } \\
\text { Salud Mental }\end{array}$ & $\begin{array}{r}156 \\
701 \\
477 \\
89 \\
150 \\
938 \\
215 \\
175\end{array}$ \\
\hline Perú & Acta Médica Peruana & 2 \\
\hline Venezuela & $\begin{array}{l}\text { Acta Gastroenterológica Latinoamericana } \\
\text { Investigación Clínica }\end{array}$ & $\begin{array}{r}10 \\
208\end{array}$ \\
\hline
\end{tabular}


ANEXO 2. Revistas que publicaron el mayor número de artículos de cada uno de los seis países estudiados, de 1973 a 1992

\begin{tabular}{|c|c|c|}
\hline \multicolumn{3}{|c|}{ Argentina } \\
\hline Revista & No. & $\%$ \\
\hline Prensa Médica Argentina & 1645 & 14,2 \\
\hline Medicina & 1297 & 11,2 \\
\hline Acta Physiologica Latinoamericana & 348 & 3,0 \\
\hline Biochimica et Biophysica Acta (Amsterdam) & 233 & 2,0 \\
\hline Acta Physiologica et Pharmacologica Latinoamericana & 195 & 1,7 \\
\hline Biochemical and Biophysical Research Communications & 101 & 0,9 \\
\hline FEBS Letters (Amsterdam) & 98 & 0,8 \\
\hline Comparative Biochemistry and Physiology [B] (Oxford) & 94 & 0,8 \\
\hline Biochemistry Journal (Londres) & 87 & 0,7 \\
\hline Molecular and Cellular Biochemistry (La Haya) & 85 & 0,7 \\
\hline Neuroendocrinology (Basilea) & 85 & 0,7 \\
\hline Otras (1110) & 7303 & 63,1 \\
\hline Total & 11571 & 100,0 \\
\hline \multicolumn{3}{|c|}{ Brasil } \\
\hline Revista & No. & $\%$ \\
\hline Biochimica et Biophysica Acta (Amsterdam) & 134 & 1,0 \\
\hline Comparative Biochemistry and Physiology [B] (Oxford) & 173 & 1,3 \\
\hline Revista Brasileira de Medicina & 1049 & 7,7 \\
\hline Revista Brasileira de Genética & 688 & 5,1 \\
\hline Brazilian Journal of Medical and Biological Research & 679 & 5,0 \\
\hline Revista do Instituto de Medicina Tropical de São Paulo & 522 & 3,9 \\
\hline Revista de Saúde Pública & 429 & 3,2 \\
\hline Arquivos Brasileiros de Psicologia & 316 & 2,3 \\
\hline Memórias do Instituto Oswaldo Cruz & 307 & 2,3 \\
\hline Revista Brasileira de Pesquisas Médicas e Biológicas & 167 & 1,2 \\
\hline Otras (1329) & 9084 & 67,1 \\
\hline Total & 13548 & 100,0 \\
\hline \multicolumn{3}{|c|}{ Chile } \\
\hline Revista & No. & $\%$ \\
\hline Biochimica et Biophysica Acta (Amsterdam) & 39 & 0,7 \\
\hline FEBS Letters (Amsterdam) & 65 & 1,1 \\
\hline Comparative Biochemistry and Physiology [B] (Oxford) & 57 & 1,0 \\
\hline Revista Médica de Chile & 2742 & 46,5 \\
\hline Archivos de Biología y Medicina Experimentales & 197 & 3,3 \\
\hline IRCS Medical Science Biochemistry & 162 & 2,7 \\
\hline Contraception (Stoneham, Massachusetts) & 52 & 0,9 \\
\hline General Pharmacology (Oxford) & 48 & 0,8 \\
\hline Biochemistry International (Sidney) & 40 & 0,7 \\
\hline Cellular and Molecular Biology (Emsford, New York) & 37 & 0,6 \\
\hline Neuroscience Letters (Limerick) & 37 & 0,6 \\
\hline Otras (691) & 2420 & 41,0 \\
\hline Total & 5896 & 100,0 \\
\hline \multicolumn{3}{|c|}{ Cuba } \\
\hline Revista & No. & $\%$ \\
\hline Revista do Instituto de Medicina Tropical de São Paulo & 9 & 1,4 \\
\hline Interferón y Biotecnología & 68 & 10,6 \\
\hline Neoplasma (Bratislava) & 36 & 5,6 \\
\hline Allergologia et Immunopathologia (Madrid) & 35 & 5,5 \\
\hline Revista Clínica Española (Madrid) & 24 & 3,8 \\
\hline Biotechnology Letters & 17 & 2,7 \\
\hline Folia Microbiologica (Praga) & 14 & 2,2 \\
\hline Acta Biotechnologica & 11 & 1,7 \\
\hline Acta Paediatrica Hungarica (Budapest) & 10 & 1,6 \\
\hline Haematologia (Budapest) & 10 & 1,6 \\
\hline Sangre (Barcelona) & 9 & 1,4 \\
\hline Acta Diabetologica Latina (Milán) & 8 & 1,3 \\
\hline
\end{tabular}


ANEXO 2. (Continuación)

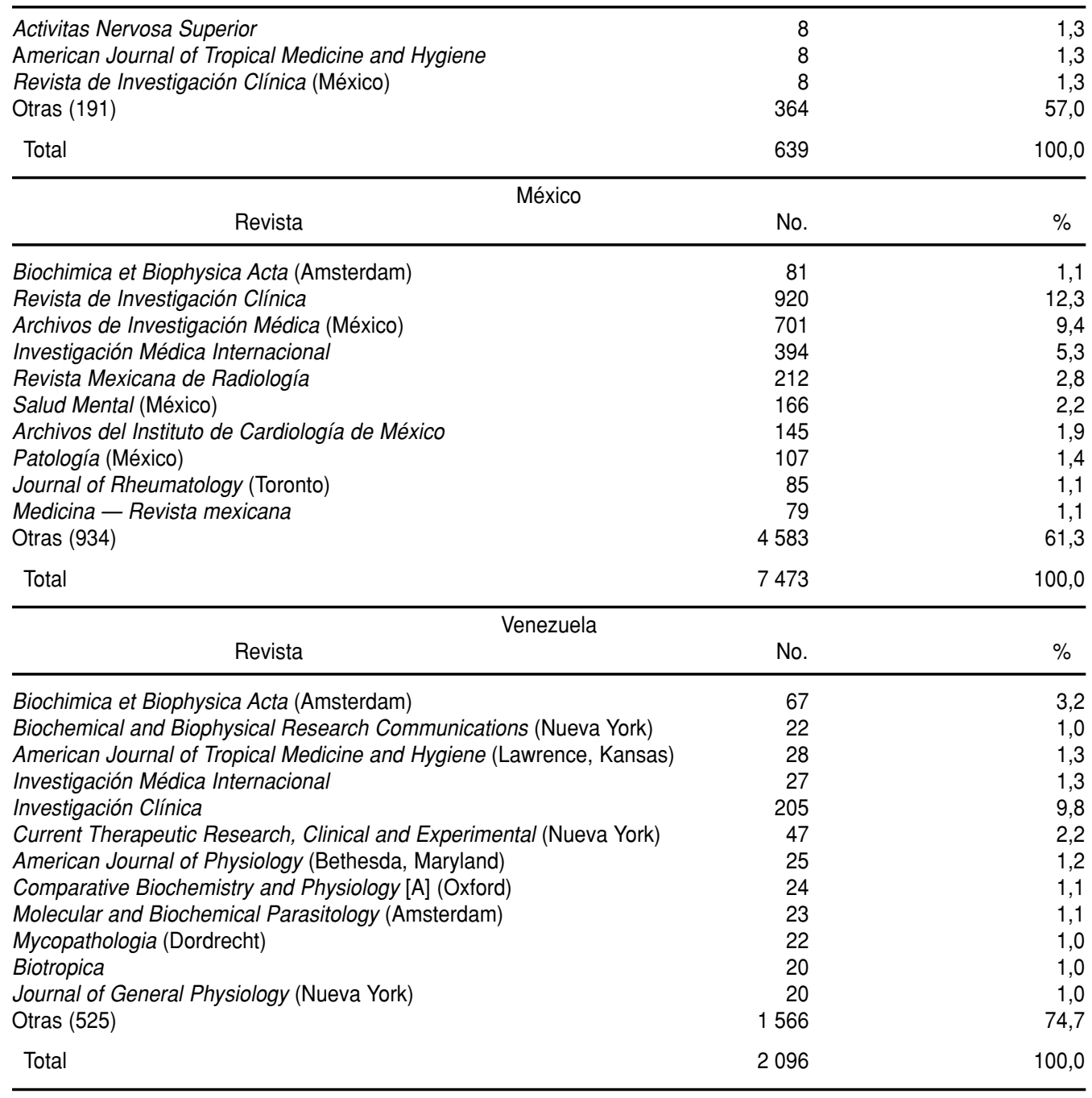

\section{REFERENCIAS}

1. Organización Panamericana de la Salud. La investigación en salud en América Latina: estudio de países seleccionados. Washington, DC: OPS; 1992. (Publicación científica 543).

2. Pellegrini A. La investigación en salud en cinco países de América Latina. Bol Oficina Sanit Panam 1993;114(2):142-157.

3. Velho L. Indicadores científicos: aspectos teóricos y metodológicos. En: E. Martínez, ed. Ciencia, tecnología y desarrollo: interrelaciones teóricas y metodológicas. Caracas: Editorial Nueva Sociedad, UNESCO; 1994.

4. Bunge M. Sociología de la ciencia. Buenos Aires: Siglo XXI; 1993.
5. Kedrov B. Acerca de las leyes del desarrollo de las ciencias. La Habana: Editorial de Ciencias Sociales; 1977.

6. Garfield E. Análisis cuantitativo de la literatura científica y sus repercusiones en la formulación de políticas científicas en América Latina y el Caribe. Bol Oficina Sanit Panam 1995;118(5): 448-456.

7. Frenk J, Bobadilla JL, Sepúlveda J, Rosenthal J, Ruelas E. Un modelo conceptual para la investigación en salud pública. Bol Oficina Sanit Panam 1986;101(5):477-489.

8. Sagasti FR, Cook C. Tiempos difíciles: ciencia y tecnología en América Latina durante el dece- nio de 80. Lima, GRADE, diciembre de 1985. (Documento fotocopiado).

9. García IC. Nuevas tendencias de la investigación biomédica y social. Washington, DC: Organización Panamericana de la Salud; 1982. (Documento fotocopiado).

Manuscrito recibido el 30 de noviembre de 1995 y aceptado para publicación en versión revisada el 20 de marzo de 1996. 
ABSTRACT The production of articles resulting from biomedical, clinical, and public health studies that originated in Argentina, Brazil, Chile, Cuba, Mexico, and Venezuela from 1973 through 1992 was analyzed to discover trends in health research in Latin America.

\section{Production of scientific} articles about health in six Latin American countries, 1973-1992
From the database of the Institute for Scientific Information (ISI), 41238 articles with first authors who resided in those countries were extracted. These articles were analyzed by subject area, type of study, country, number of authors and institutions that participated in the investigation, and citations received by each article. Also analyzed were 95 articles in epidemiology selected from a pool of 570 published by authors from the six countries in 11 public health journals that enjoy international prestige.

The results showed that the number of published works increased by $117 \%$ between the first and last five-year periods within the study period. Clinical research was distributed the most evenly among the countries, and public health research was the most concentrated $(60.7 \%$ originated in Brazil). The numbers of biomedical and public health research articles showed relatively more growth than those reporting on clinical research throughout the period. A relative decrease was found in articles by only one author, which suggests a greater frequency of team efforts, and an increase was seen in articles with authors tied to two or more national or foreign institutions, which indicates greater cooperation between institutions and countries. The average number of citations received by each article was 3, which was less than half the number received by the articles in the ISI database (7.78). Regarding the subset of 95 articles in epidemiology, the great majority (96\%) dealt with infectious diseases or maternal and child health, while in the international literature $78 \%$ of such articles were about chronic diseases. This group of articles gave evidence of more cooperation with international institutions and had a citation index of 4.36 per article.

It is concluded that, despite the inherent limitations, this type of study reveals some general trends in the development of research in the six Latin American countries with the greatest scientific production and makes it possible to formulate hypotheses on the factors that influence these trends. Taken with the proper caution, the results of studies like this one can be of great value in defining health science and technology policies. 